\title{
Glioma Marker Network
}

National Cancer Institute

\section{Source}

National Cancer Institute. Glioma Marker Network. NCI Thesaurus. Code C19669.

A group of of six collaborating laboratories at medical centers located throughout the U.S. working to identify new molecular alterations that may be useful for brain tumor diagnosis. 